\title{
Microwave Class-E Power Amplifiers
}

\author{
Zoya Popović ${ }^{1}$ and José A. García ${ }^{2}$ \\ ${ }^{1}$ Department of Electrical, Computer and Energy Engineering, University of Colorado, Boulder, CO, 80309, U.S.A. \\ ${ }^{2}$ Department of Communications Engineering, University of Cantabria, 39005 Santander, Spain
}

\begin{abstract}
This paper reviews circuit architectures and demonstrated class-E power amplifiers in the UHF and microwave frequency range. Scaling class-E soft-switching operation to high frequencies presents a number of challenges, particularly in the control of parasitic reactances of the device and the circuit. Different approaches have been taken, from using parasitics of lumped elements to provide the correct fundamental and harmonic impedances in the UHF range, to transmission-line implementations at frequencies above $10 \mathrm{GHz}$.
\end{abstract}

Index Terms - power amplifiers, class-E, soft switching, supply modulation

\section{Class-E AmPLifier FREQUENCY SCALING}

Since Nathan Sokal's invention of the class-E power amplifier (PA), the vast majority of class-E results have been reported at $\mathrm{kHz}$ and $\mathrm{MHz}$ frequencies, but the concept is increasingly applied in the UHF [1-3], microwave, e.g. [4-10], and millimeter-wave range, e.g. [11]. The focus of this paper is a brief review of some interesting concepts in highfrequency class-E PAs and related circuits.

The well-known ideal theoretical maximum frequency of class-E operation is given by:

$$
f_{\text {max }, E} \cong I_{\max } /\left(56.5 C_{\text {OUT }} V_{d s}\right),
$$

where the transistor output capacitance $C_{\text {OUT }}$ limits the frequency range of class-E operation. $C_{O U T}$ can be de-embedded and used as a part of the output matching circuit, which has a fundamental impedance of

$$
Z_{E}=0.28 /\left(\omega C_{O U T}\right) e^{j 49^{\circ}}
$$

and all higher harmonics are open-circuited. These equations are derived under a number of assumptions, including a high Q-factor of the output circuit. At lower frequencies, these impedance conditions can be implemented with lumped elements, but in the microwave frequency range distributed elements have lower loss and become sufficiently small. Fig. 1a shows a transmission-line implementation with only the second harmonic termination [1].

At higher frequencies, it is difficult to achieve optimal class-E operation associated with soft switching, since the switching frequency in Fig. $1 \mathrm{a}$ is at least 5 times lower than the $f_{T}$ of the switching device, and "on" and "off" resistances are finite, resulting in an efficiency drop [4]. Although ideally all harmonics of the switching frequency are open-circuited, it is often sufficient to terminate only the $2^{\text {nd }}$ harmonic, since device gain drops and circuit losses increase to a point of diminishing returns. Voltage waveforms for the ideal case corresponding to the circuit in Fig.1a are shown in Fig.1b. Active device internal parasitics are substantial at high frequencies and difficult to de-embed from nonlinear models, so the design of class-E waveforms at the virtual drain, where they are specified, becomes a challenge. Additionally, the nonlinearity of the output capacitance affects the voltage and current timedomain waveforms, and increases the voltage or current stress on the device and lowers the output power, as shown in Fig.1c. Another limitation is the device breakdown voltage, which needs to be 3.56 times the supply voltage for class-E operation, and therefore at high frequencies typically the output power is reduced. Design challenges also include input matching (switch control signal), bias line design, and losses in the matching network.

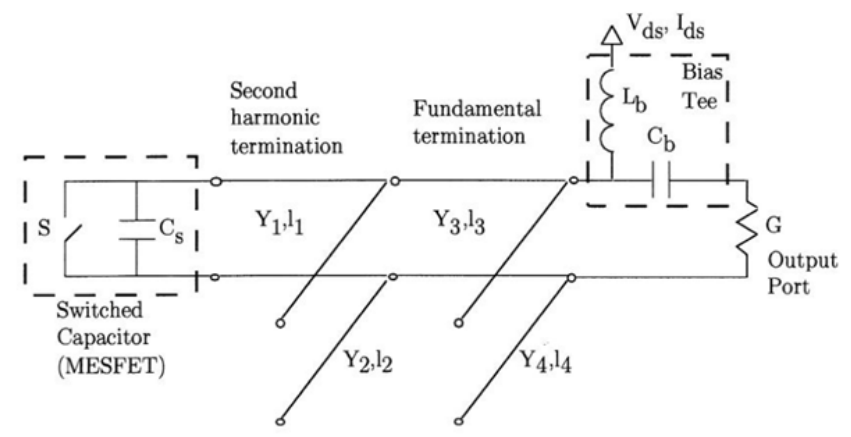

(a)

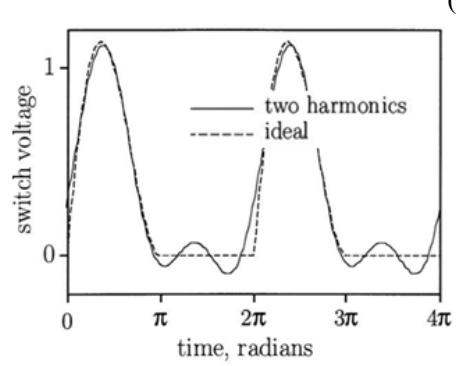

(b)

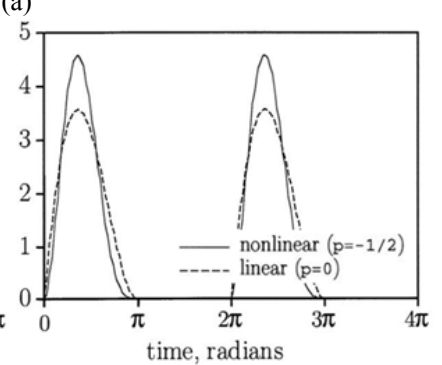

(c)
Fig. 1. (a) Transmission-line Class-E circuit topology for high-frequency class-E PAs with only $2^{\text {nd }}$ harmonic open-circuited, and (b) associated switch voltage waveform degradation. (c) When the device output capacitance is nonlinear, the voltage peak increases theoretically $28 \%$ for a simple squareroot nonlinearity [4]

\section{LUMPED-ELEMENT UHF CLASS-E PAS}

The standard series resonant circuit at the output of a classE PA, as proposed by Sokal, requires a high loaded Q-factor, but at UHF and low microwave frequencies, the parasitics associated with a high inductance value may result in self- 
resonance below the most significant higher order harmonics to be properly terminated. A parallel resonant circuit, tuned to provide the open condition at the second, third harmonic or any convenient frequency between them, is shown in Fig.2 and is discussed in [12] and [13]. A network that assures an impedance closer to the optimum for both $2^{\text {nd }}$ and $3^{\text {rd }}$ harmonic implements the parallel resonance with the coil parasitic capacitance [3]. If the value is reduced below the one that selfresonates between $2 f$ and $3 f$, its resistive losses may be reduced. The resulting lower Q-factor at the fundamental widens the bandwidth. The output network is completed with a series reactance and a shunt capacitor to provide the class-E impedance at the fundamental.

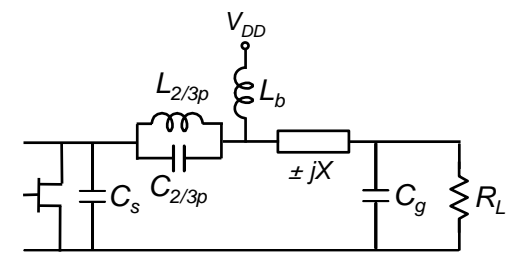

Fig. 2. Lumped-element class-E PA topology [13]. The RF choke position is chosen to reduce the impact of its parasitics on the desired drain impedance.

Alternative lumped-element implementations, e.g. [3], are derived from the frequency-domain transmission-line synthesis approach illustrated in Fig.1. The use of coils and capacitors is also reported at UHF for other ZVS and ZVDS cases in the continuum of Class-E modes [14].

A lumped-element UHF implementation example based on a CGH35030F GaN HEMT from Wolfspeed is shown in Fig.3. The Class-E operation is approximated over a wide bandwidth, trading resonant circuit Q-factors at the fundamental and $2^{\text {nd }} / 3^{\text {rd }}$ harmonics. A peak efficiency of $85.7 \%$ is measured, and maintained above $80 \%$ over a $230 \mathrm{MHz}$ frequency range ( $27 \%$ fractional bandwidth).
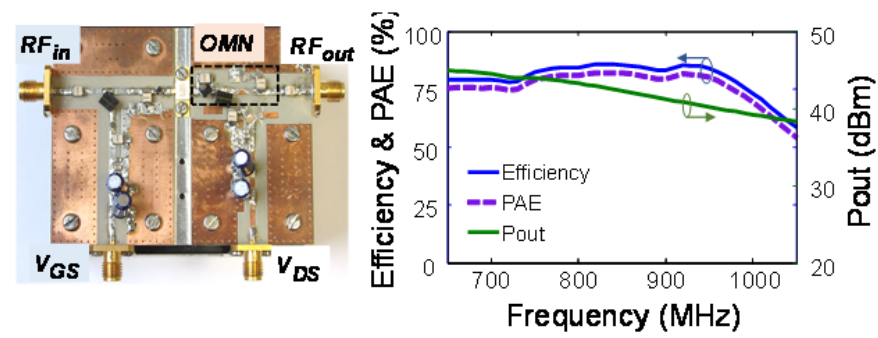

Fig. 3. Lumped-element class-E PA at UHF with measured results.

The first transmission-line implementations at $500 \mathrm{MHz}$, $1 \mathrm{GHz}$ and $2 \mathrm{GHz}$ using a GaAs MESFET (CLY5) are shown in Fig. 4 [1]. In this work, the theoretical degradation with increasing frequency for a given device is shown experimentally, and the circuits are designed analytically for first-pass success. Above $1 \mathrm{GHz}$, the device parameters do not satisfy Eq. (1) and the mode of operation first becomes a sub-optimal class-E mode, degrading into $\mathrm{AB}$ as the frequency increases further. The output power could not be well predicted due to the inadequacy of non-linear models in 1995. A discussion on frequency scaling up to X-band is also presented in [15] and shown on GaAs MMIC and GaN hybrid example PAs.

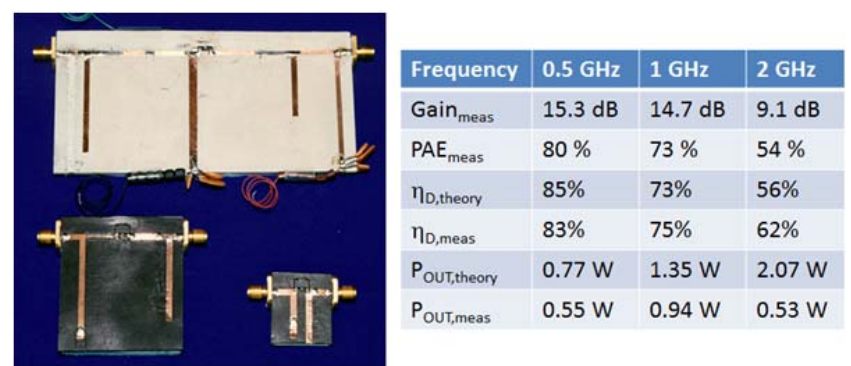

Fig. 4. Left: Photograph of first reported transmission-line $0.5,1$ and $2 \mathrm{GHz}$ class-E PAs implemented with the CLY5 GaAs MESFET [1]. Right: measured and theoretically predicted performance.

\section{MicRowAVE CLASS-E AMPLIFIERS}

A number of hybrid class-E amplifiers were reported at Cband, e.g. [4] and X-band [5-10] with GaAs FETs. MMIC class-E PAs are demonstrated in GaAs [5,15], InP [6], GaN [9] and CMOS [11,16]. In [4], a FLK052WG class-E microstrip PA that delivers $0.61 \mathrm{~W}$ with a compressed gain of 9.8 $\mathrm{dB}$, a drain efficiency of $81 \%$, and a PAE of $72 \%$ at $5 \mathrm{GHz}$ is integrated in a spatial combining array. Anti-resonant slot antennas are used to present the harmonic terminations. Several X-band GaAs class-E PAs are demonstrated with PAE $>60 \%$, and an example is shown in Fig.5, where a class-E PA stage is incorporated into a two-stage PA with the first stage also operated in class-E mode, reaching a total $\mathrm{PAE}=52 \%$ with the drain efficiency of the second stage of $62 \%$ [6].

Fig. 6 shows a reconfigurable $10-\mathrm{GHz}$ PA with MEMS ohmic switch output matching network which enables the PA to operate in either linear class-A/AB or high-efficiency classE. The insertion loss of the matching network in different states is below $0.3 \mathrm{~dB}$, causing a few points degradation in PAE compared to a non-reconfigurable static PA made on the same substrate and with the same GaAs die [8].
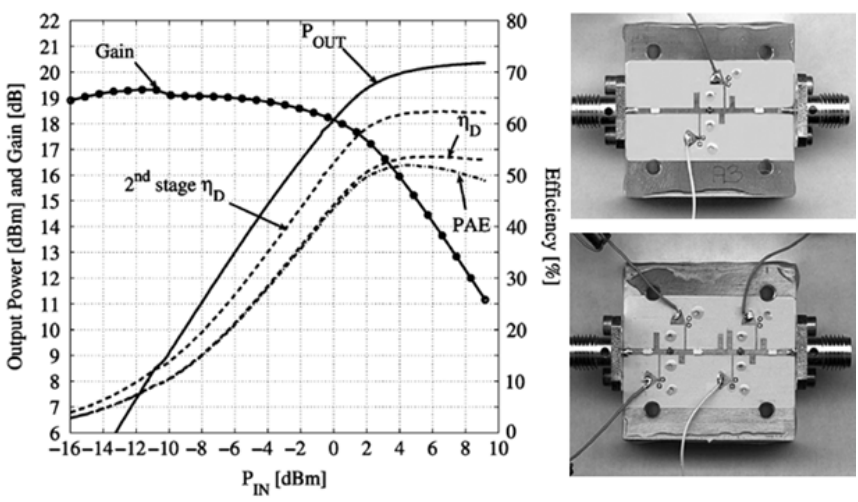

Fig. 5. Photographs of the output stage only (top right) of a hybrid class-E 10$\mathrm{GHz}$ GaAs PA with PAE $=62 \%$, and a two-stage class-E PA with both stages operating close to class-E (bottom). The plots show measured data for the two-stage PA calibrated at the SMA connectors [6]. 
One of the first reported GaAs class-E X-band PAs is discussed in [5] and a photograph of the single-stage MMIC and its measured performance is shown in Fig.7, showing $\mathrm{PAE}=65 \%$ at a power of $24 \mathrm{dBm}$.
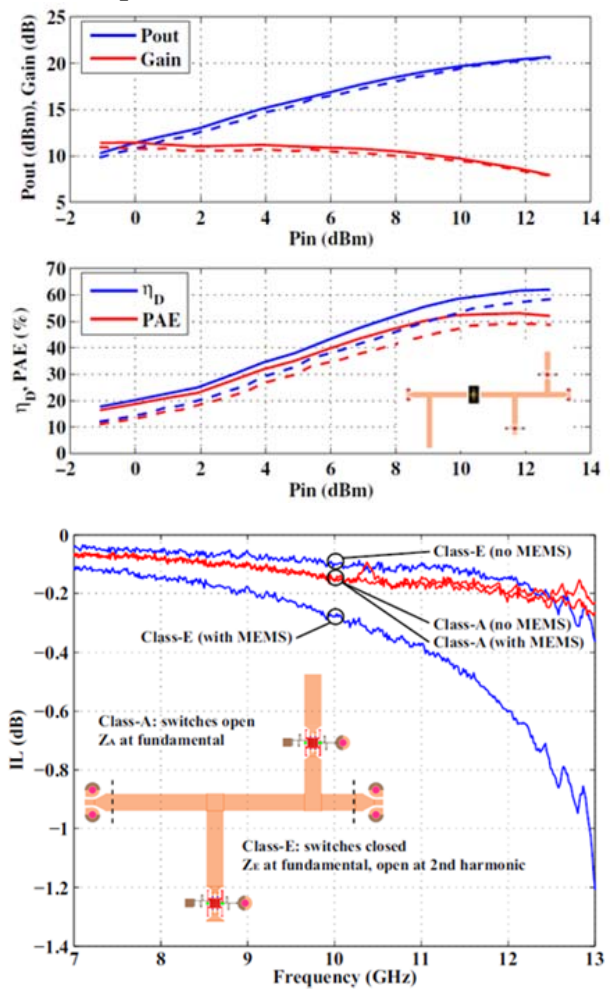

Fig. 6. Top: Measured data on a $10-\mathrm{GHz}$ mode-reconfigurable PA that uses MEMS switches to control the output matching circuit between a linear class$A$ and an efficient class-E [8]. The dashed lines show efficiency loss due to the reconfigurable MEMS network by comparing to a static class-E PA. Bottom: Measured insertion loss for the class-A and class-E matching network shows a degradation of at most $0.2 \mathrm{~dB}$ at $10 \mathrm{GHz}$.

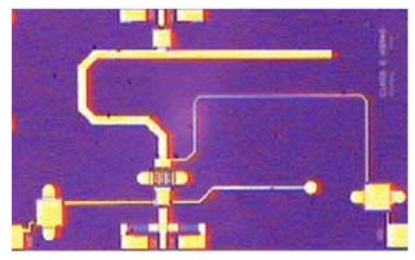

$1.5 \mathrm{~mm} \times 0.6 \mathrm{~mm}$ GaN MMIC

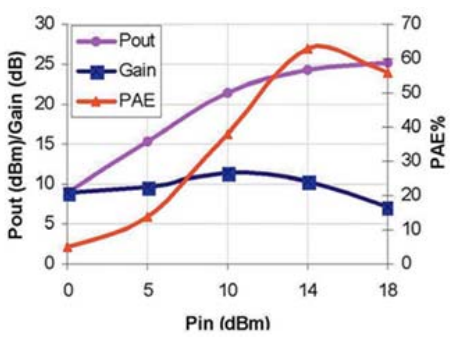

Fig. 7. GaAs MMIC PA from [5] measured at 10.6GHz.

MMIC integrated class-E PAs at X-band include an InP two-stage amplifier shown in Fig.8 [6], which had a measured $\mathrm{PAE}=52 \%$ and compared well with the hybrid GaAs version in [6] in terms of efficiency points lost due to the first stage. In the case of two-stage class-E PAs [7], the interstage network can be designed to provide input harmonic wave-shaping for a more squared waveform that controls the transistor operating as a switch.

More recently, several CMOS class-E PAs have been reported, where stacking of devices is used to overcome the breakdown voltage limitation. In [11], a CMOS IC at $93 \mathrm{GHz}$ shows PAE $>40 \%$, demonstrating that Sokal's class-E concept can be extended to extremely high frequencies, Fig. 9.
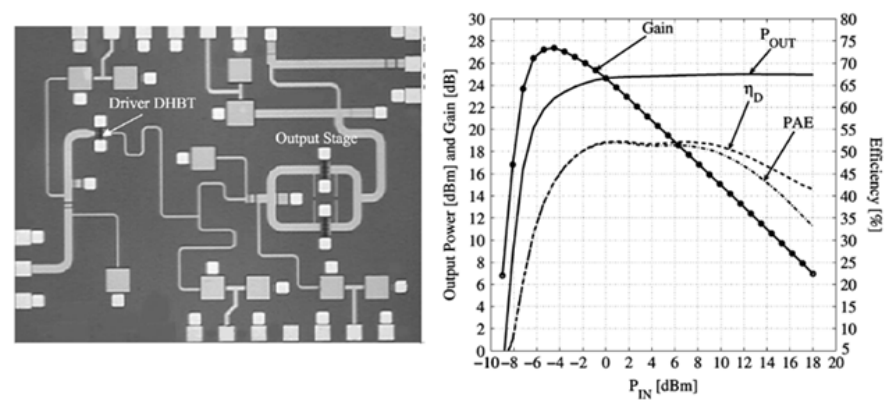

Fig. 8. InP two-stage PA MMIC photo (left) and measured performance (right) [6]. The MMIC is $2.65 \mathrm{~mm} \times 2.1 \mathrm{~mm}$ in size.
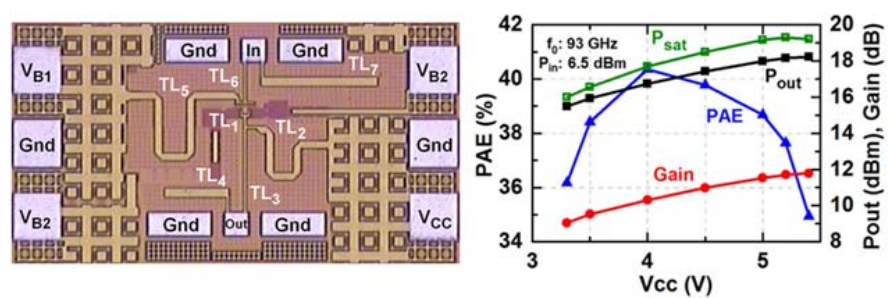

Fig. 9. Bi-CMOS W-band class-E MMIC photo and performance [11].

\section{Class-E PAS FOR High PAPR Signal TRANSMitTERS}

Ideal class-E equations detailed in, e.g. $[4,10]$, show that the output power across a fixed load is proportional to the square of the drain (collector) voltage:

$$
P_{\text {OUT }}=0.5 R_{E}\left(1.86 \pi \omega C_{O U T}\right)^{2} V_{D D}^{2},
$$

which in turn implies that class-E PAs are very well suited to envelope tracking for efficiency improvement when the signal has a high peak-to-average ratio [17]. Tracking using a class-E PA and a class-E dc-dc converter with excellent efficiencies in the 1-GHz range is shown in [18-19]. The linearity of such an envelope-tracked transmitter is analyzed experimentally in [10] at a $10 \mathrm{GHz}$ carrier with a two-tone signal. A two-stage $150-\mathrm{nm} \mathrm{GaN}$ on SiC PA is demonstrated with the output stage designed using class-E design rules, with a power over $10 \mathrm{~W}$, Gsat $>20 \mathrm{~dB}$ and peak PAE $>60 \%$ [9] where the efficiency remains above $50 \%$ at $10 \mathrm{~dB}$ back-off. This PA was specifically designed for supply modulation, with efficiency curves shown in Fig.10. Another method for increasing efficiency for high PAPR signals is outphasing, and it has been shown that classE PAs lend themselves easily to a non-isolated (Chireix) architecture [2].
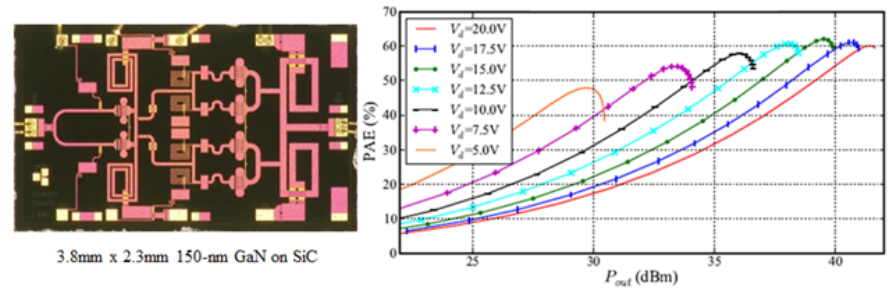

Fig. 10. 10-GHz class-E MMIC PA for supply modulation [9]. 
The class-E PA is classically a tuned topology, which in practice has about $10 \%$ bandwidth over which the frequency, power and PAE remain close to the maximum. Extending the bandwidth of class-E PAs by matching circuit design is investigated in e.g. [20-22]. In [20], over $80 \%$ efficiency is obtained in a low-power class-E PAE from $1.7-2.7 \mathrm{GHz}$ by input matching that includes second-harmonic optimization. In [21], the PAE exceeds $63 \%$ over the $0.9-2.2 \mathrm{GHz}$ band with $3-\mathrm{dB}$ variation in output power. In [22], single-ended and differential matching networks are investigated to obtain a $1.7-2.2 \mathrm{GHz}$ operational bandwidth of a class-E PA implemented in 90-nm CMOS operating in sub-optimal mode with efficiency over $42 \%$ and output power above $25 \mathrm{dBm}$.

\section{CONCLUSIONS}

Other diverse aspects of class-E microwave circuits include oscillators, rectifiers and dc-dc converters. [23] examines stability of class-E PAs, while [24] discusses effects of parameter tolerances on class-E behavior. Spatial power combining of 16 or more class-E PAs is demonstrated in [25]. Fig. 11 shows the circuit side of an example 10-GHz array, where the 16 PAs are fed with a corporate Wilkinson combiner feed network, while the outputs are combined in free space upon radiation from a 16-element in-phase fed patch antenna array. The freespace combining efficiency is estimated to be $80 \%$, which is higher than a 4-level corporate network at $10 \mathrm{GHz}$. In summary, this paper presents a very brief overview that only gives a glimpse into the vast area of microwave class-E PAs which resulted from Nathan Sokal's pioneering work.
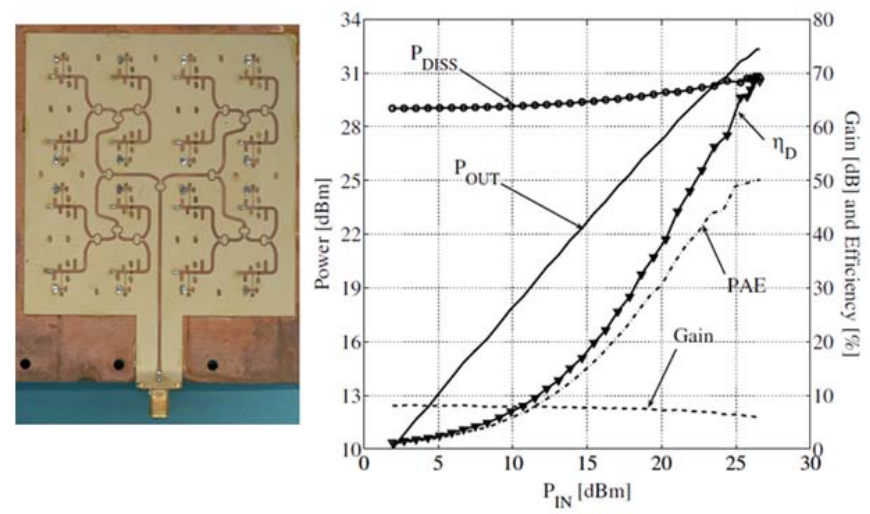

Fig.11. Spatial power combiner with class-E PAs at $10 \mathrm{GHz}$ [20].

\section{REFERENCES}

[1] T. Mader, Z. Popovic, "The transmission-line high-efficiency class-E amplifier," IEEE Guided Wave Lett., vol.5 ,pp. 290-292, Sept.1995.

[2] R. Beltran, F. H. Raab and A. Velazquez, "HF outphasing transmitter using class-E power amplifiers," 2009 IEEE MTT-S International Microwave Symposium Digest, Boston, MA, 2009, pp. 757-760.

[3] J. A. Garcia, R. Marante and M. de las Nieves Ruiz Lavin, "GaN HEMT Class-E Resonant Topologies for UHF DC/DC Power Conversion," in IEEE Transactions on Microwave Theory and Techniques, vol. 60, no. 12, pp. 4220-4229, Dec. 2012.

[4] T. B. Mader, E. W. Bryerton, M. Markovic, M. Forman and Z. Popovic, "Switched-mode high-efficiency microwave power amplifiers in a free- space power-combiner array," IEEE Transactions on Microwave Theory and Techniques, vol. 46, no. 10, pp. 1391-1398, Oct 1998.

[5] R. Tayrani, "A monolithic X-band class-E power amplifier, $23^{\text {rd }} I E E E$ GaAs IC Symp Digest 2001, Baltimore, MD, USA, 2001, pp. 205-208.

[6] S. Pajic, N. Wang, P. Watson, T. Quach, Z. Popovic, "X-band two-stage high-efficiency switched-mode power amplifiers," IEEE Trans. Microwave Theory Techn., vol. 53, no. 9, pp. 2899-2907, Sept. 2005.

[7] Tayrani, Reza. "Two stage microwave Class E power amplifier." U.S. Patent No. 7,265,619. 4 Sep. 2007.

[8] P. J. Bell, Z. Popovic and C. W. Dyck, "MEMS-switched class-A-to-E reconfigurable power amplifier," 2006 IEEE Radio and Wireless Symposium, 2006, pp. 243-246.

[9] S. Schafer, M. Litchfield, A. Zai, Z. Popovíc and C. Campbell, "X-band MMIC GaN power amplifiers designed for high-efficiency supplymodulated transmitters," 2013 IEEE MTT-S International Microwave Symposium Digest (MTT), Seattle, WA, 2013, pp. 1-3.

[10] N. Wang, X. Peng, V. Yousefzadeh, D. Maksimovic, S. Pajic and Z. Popovic, "Linearity of X-band class-E power amplifiers in EER operation," IEEE Transactions on Microwave Theory and Techniques, vol. 53, no. 3, pp. 1096-1102, March 2005.

[11] P. Song, M. Oakley, A. C. Ulusoy, M. Kaynak, B. Tillack, G. Sadowy, J. Cressler, "A Class-E Tuned W-Band SiGe Power Amplifier With 40.4\% Power-Added Efficiency at $93 \mathrm{GHz}$," in IEEE Microwave and Wireless Components Letters, vol. 25, no. 10, pp. 663-665, Oct. 2015.

[12] M. Iwadare, S. Mori, "Even-harmonic resonant class E tuned amplifier without RF choke," Electronics and communications in Japan, Part 1, Vol. 79, No. 1, 1996.

[13] R. Negra and W. Bachtold, "Lumped-element load-network design for class-E PAs," IEEE Trans. Microw. Theory Techn., vol. 54, No. 6., pp. 2684-2690, Jun. 2006.

[14] J. Cumana, A. Grebennikov, G. Sun, N. Kumar and R. H. Jansen, "An Extended Topology of Parallel-Circuit Class-E Power Amplifier to Account for Larger Output Capacitances," IEEE Transactions on Microwave Theory and Techniques, vol. 59, no. 12, pp. 3174-3183, Dec. 2011.

[15] P. Colantonio et al., "High frequency class E design methodologies," European GaAs/Semiconductor Application Symp., GAAS 2005, Paris, 2005, pp. 329-332.

[16] D. Y. C. Lie et al., "Highly Efficient Monolithic Class E SiGe Power Amplifier Design at 900 and $2400 \mathrm{MHz}$," in IEEE Transactions on Circuits and Systems I: Regular Papers, vol. 56, pp. 1455-1466, July 2009.

[17] M. Weiss, F. Raab, Z. Popovic, "Linearity of X-band class-F power amplifiers in high-efficiency transmitters," IEEE Trans. Microwave Theory Techn., vol. 49, no. 6, pp. 1174-1179, Jun 2001.

[18] J. A. García, R. Marante, M. N. Ruiz and G. Hernández, "A $1 \mathrm{GHz}$ frequency-controlled class $\mathrm{E}^{2} \mathrm{DC} / \mathrm{DC}$ converter for efficiently handling wideband signal envelopes," 2013 IEEE MTT-S International Microwave Symposium Digest (MTT), Seattle, WA, 2013, pp. 1-4.

[19] M. N. Ruiz, A. L. Benito, J. R. Pérez-Cisneros, P. L. Gilabert, G. Montoro and J. A. García, "Constant-gain envelope tracking in a UHF outphasing transmitter based on continuous-mode class-E GaN HEMT PAs," 2016 IEEE MTT-S International Microwave Symposium (IMS), San Francisco, CA, 2016, pp. 1-4.

[20] V. Chaudhary and I. S. Rao, "A novel $2 \mathrm{GHz}$ highly efficiency improved class-E Power Amplifier for Base stations," 2015 International Conference on Communications and Signal Processing (ICCSP), Melmaruvathur, 2015, pp. 0940-0944.

[21] K. Chen and D. Peroulis, "Design of Highly Efficient Broadband ClassE Power Amplifier Using Synthesized Low-Pass Matching Networks," IEEE Trans. Microwave Theory Techn., pp. 3162-3173, Dec. 2011.

[22] M. D. Wei, D. Kalim, D. Erguvan, S. F. Chang and R. Negra, "Investigation of Wideband Load Transformation Networks for Class-E Switching-Mode Power Amplifiers," IEEE Trans. Microwave Theory and Techniques, vol. 60, no. 6, pp. 1916-1927, June 2012.

[23] J. de Cos, A. Suárez and J. A. García, "Hysteresis and Oscillation in High-Efficiency Power Amplifiers," IEEE Transactions on Microwave Theory and Techniques, vol. 63, no. 12, pp. 4284-4296, Dec. 2015.

[24] F. H. Raab, "Effects of circuit variations on the class $E$ tuned power amplifier," IEEE Journal of Solid-State Circuits, vol. 13, no. 2pp. 239247, Apr 1978.

[25] S. Pajic, Z. Popovic, "An efficient X-band 16-element spatial combiner of switched-mode power amplifiers," IEEE Trans. Microwave Theory Techn., pp. 1863-1870, July 2003. 